\title{
Managementinformation
}

Michael Reiss*

\section{Komplexe Push-Pull-Systeme: Ein integriertes Rahmenkonzept zur informationellen Steuerung von Wertschöpfungsprozessen}

DOI 10.1515/iwp-2016-0022

Zusammenfassung: Push- und Pull-Informationen fungieren als initialisierende Steuerungsimpulse sowohl für strategische Innovationsprozesse (z. B. Knowledge Push-Innovationen) als auch operative Versorgungsprozesse (z.B. On-Demand-Systeme). Vorhandene Ansätze liefern eine suboptimale Performanz, weil sie mehrere Facetten der Wertschöpfungs- und Steuerungskomplexität ausklammern. Abhilfe kann hier ein komplexer Push-Pull-Ansatz schaffen. Performanz-Verbesserungen lassen sich vor allem mithilfe von Multi-Impuls-Modellen und einer hybriden Involvierung von Wertschöpfungsakteuren erzielen.

Deskriptoren: Innovation, Management, Pull-Steuerung, Push-Steuerung, Auftragssteuerung

Complex push-pull-systems: an integrated framework for the informational control of value added processes

Abstract: Push and pull information serve as triggers for strategic innovation processes (e. g. knowledge-push innovations) as well as operational supply processes (e.g. ondemand systems). Existing approaches provide a suboptimal performance since they exclude several facets of the complexity of both value adding and control. A complex push-pull approach provides better performance. Performance improvements can be achieved by using multi-trigger models and a hybrid mode of involving stakeholders into value added-systems.

Descriptors: innovation, management, push control, pull control, operations management

Systèmes complexes de push-pull: un concept-cadre intégré à la gestion informationnelle des processus de création de valeur

*Kontaktperson: Prof. Dr. Michael Reiss, Lehrstuhl für Organisation, Universität Stuttgart, Keplerstraße 17, 70174 Stuttgart,

E-Mail: michael.reiss@bwi.uni-stuttgart.de
Résumé: Les informations "push » et "pull " servent d' impulsions à l' initialisation des processus d'innovation stratégique (innovations de « technology push » par exemple) ainsi que des processus de logistique opérationnelle (par exemple les systèmes à la demande). Les approches existantes n' offrent pas une performance optimale parce qu'elles excluent plusieurs facettes de la complexité à la fois dans le processus de création de valeur et dans le contrôle. Une approche complexe peut aider ici. Une amélioration de la performance est possible en utilisant des modèles multi-impulsion et un rôle hybride joué par les acteurs de création de valeur.

Descripteurs: gestion, innovation, modèle push-pull, gestion des opérations

\section{Problemstellung: Komplexität der Wertschöpfungssteuerung}

Sowohl operative Versorgungsprozesse der Produktion, der Logistik und des Vertriebs als auch strategische Innovationsprozesse für Produkt-, Prozess- oder Geschäftsinnovationen (Tidd; Bessant 2013) werden durch spezifische Steuerungsinformationen initiiert. Hierzu kommen einerseits angebotsseitige Impulse zum Einsatz. $\mathrm{Zu}$ diesen so genannten Push-Informationen zählen etwa Patente oder Lieferzusagen. Andererseits beruht eine Initialisierung von Wertschöpfungsprozessen auf Pull-Informationen in Gestalt von Bedarfen, Kundenaufträgen oder Calls im Rahmen von Ausschreibungen. Umstritten ist allerdings der Stellenwert dieser Impulse für den Erfolg von Wertschöpfungsprozessen: Häufig kommt der angestrebte Fit von Bereitstellung und Bedarf, das Kriterien für eine erfolgreiche Wertschöpfung, nicht zustande, nur verzögert zustande oder ist mit hohen Kosten verbunden. In solchen Fällen treten Misfits in Gestalt von Flops, Unterversorgung (z. B. Fehlmengen, Verzögerungen, schlechter Servicegrad) oder Verschwendung (z.B. hohe Lagerbestände, Blindleistung) auf. Plakativ werden einige dieser Diskre- 
panzen beispielsweise als „Pflegenotstand“, „Servicewüste“ oder „Rattenrennen“ (Wettlauf von Technologieunternehmen ohne Orientierung am Kundennutzen) charakterisiert. Die gängigen Erklärungsansätze für fehlgeschlagene Innovationsprozesse oder schlechte Versorgung fokussieren meist zu schwach ausgeprägte Pushoder Pull-Impulse. Häufig wird die Impulsschwäche durch eine unzureichende Informationsbasis für die Management-Aktivitäten verursacht, vor allem hinsichtlich Fehlerfreiheit, Objektivität, Glaubwürdigkeit und Aktualität (Rohweder et al. 2008). Deutlich seltener werden inkompatible Impulse oder suboptimale Kopplungen von Pushund/oder Pull-Impulsen für den Misserfolg verantwortlich gemacht.

Allerdings weisen diese Erklärungsansätze selbst insofern Schwachstellen auf, als sie mehrere Dimensionen der Komplexität von Steuerungs- und Wertschöpfungskonstellationen nicht erfassen können. Mithilfe der herkömmlichen Einfach-Architekturen kann man beispielsweise nicht erklären, warum einerseits Bauprojekte häufig mit großer Verspätung abgeschlossen werden oder Innovationen auf den Gebieten „Intelligentes Haus“, E-Mobilität und barrierefreie Kommunikation wenig erfolgreich verlaufen, während andere innovative Wertschöpfungsprozesse wie z.B. Leasing, E-Commerce, Wikipedia oder mobile Web-Services sehr erfolgreich sind.

Daraus resultiert die Forderung nach komplexitätsgerechten Push-Pull-Ansätzen. Deren Gestaltung wird in drei Etappen bewerkstelligt: Aufbauend auf einer Abklärung der Modellierung von Steuerung und Wertschöpfung erfolgen eine Analyse des Status-Quo der vorhandenen Push-Pull-Ansätze und deren Schwachstellen in puncto Komplexitätshandhabung. Schließlich werden zur Behebung dieser Defizite komplexitätsgerechte Modell-Ansätze konzipiert, die vor allem die unterschiedlichen Ebenen der Wertschöpfung, die Relevanz zusätzlicher Kategorien von Wertschöpfungsakteuren, die Einbindung von Wertschöpfungsakteuren mit hybriden Rollen sowie das Zusammenspiel multipler Impulse erfassen. Aufgabe eines Ausblicks ist es, Szenarien für die erforderliche Weiterentwicklung des Ansatzes zu skizzieren, um verbleibende Schwachstellen zu beseitigen.

\section{Architektur der Wertschöpfungssteuerung}

\subsection{Operative und strategische Push-Pull- Steuerung}

„Push“ und „Pull“ fungieren als initialisierende informatorische Impulse für die Steuerung von Wertschöpfungsprozessen. Zum einen finden sich solche Modelle im operativen Versorgungsmanagement, wo Produktions- und Logistikvorhaben über die Wertschöpfungsstufen gezogen (PullPrinzip) oder geschoben (Push-Prinzip) werden. Hier sind sie eingebettet in Informationssysteme der MaterialflussSteuerung, z. B. in Just-in-Time-Systeme (Simchi-Levi et al. 2008). Zum anderen initialisieren diese Informationen die strategisch ausgerichteten Innovationsprozesse, etwa in Gestalt von Market- bzw. Demand-Pull-Innovationen oder Supply-, Technology-, Resource- bzw. Knowlegde-Push-Innovationen (Di Stefano; Gambardella; Verona 2015).

Bei Pull-Informationen handelt es sich um Problemstellungen, etwa Bedarfe, Anfragen, Calls (bei Ausschreibungen und Konzeptwettbewerben), Pflichtenhefte, Bestellungen, Aufträge, Beschwerden, Mahnungen, Änderungswünsche und vertraglich vereinbarte Leistungsverpflichtungen. Hingegen haben Push-Informationen den Charakter von Problemlösungen, etwa in Gestalt von Angeboten, Produktpräsentationen auf Messen, Erfahrungswerten, kapazitätsseitig abgeklärte Liefermengen, Ideen, Know-how, Patenten, Submissionen bei Ausschreibungen von Entwicklungs- oder Produktionsaufträgen sowie vertraglich geregelten Leistungszusagen (z. B. in Service Level Agreements).

Ganz allgemein bezeichnen „Push“ und „Pull“ unterschiedliche Auslöser von Veränderungen, Wandel und Bewegungen über Druck (Push) oder über Sog (Pull). Zu solchen Push-Faktoren aus dem Kontext von Wertschöpfungssystemen zählen z. B. Gesetzesänderungen, der Klimawandel, die Energiewende, Epidemien oder ComputerViren. Die Palette der Pull-Faktoren enthält beispielweise den Veränderungssog durch Megatrends wie die Internationalisierung oder Digitalisierung. Häufig repräsentieren also die Pull- oder Pull-Impulse für Wertschöpfungsprozesse (z. B. Anti-Viren-Programme) nicht den absoluten Ursprung eines Prozesses, sondern lediglich Reaktionen auf exogene Kontextveränderungen. Keinen Bezug zu den Bausteinen „Problemstellung“ oder „Problemlösung“ in Wertschöpfungsprozessen haben etwa vorliegende Pushoder Pull-Ansätze im Sinne unterschiedlicher Strategien zur Mobilisierung des Leistungspotenzials von Humanressourcen (Hagel; Brown 2005). 


\subsection{Modellierungen von Wertschöpfungssystemen}

Die Modelle eines Wertschöpfungssystems setzen sich aus drei Bausteinen zusammen: (1) Wertschöpfungsresultat, wie beispielsweise ein innovatives Produkt oder der rechtzeitige Zugriff auf Daten. (2) Wertschöpfungsprozesse, z. B. Software-Entwicklung oder das personalisierte Angebot z.B. von TV-Inhalten im Rahmen des interaktiven Fernsehens. (3) Wertschöpfungspotenziale: Hierzu zählen die Prozesstechnologien, die Manpower-Ausstattung sowie Koordinationsvorkehrungen, z.B. Spielregeln, Gesetze und Koordinationsorgane. Diese Infrastrukturen übernehmen die Rolle von Katalysatoren, „Facilitators“ oder „Enablers“ von Wertschöpfungsprozessen: So ermöglichen digitale Tools der Produktions- und Logistiksteuerung (z. B. RFID, Simulationstechniken) und des Kundenbeziehungsmanagements (etwa Online-Konfiguratoren oder digitale Zahlungsmittel) beispielsweise On-Demand-Geschäfte, die virtuelle Zusammenarbeit in einem Team ,around the clock and the globe“, telemedizinische Services sowie die profitable Bedienung von Aufträgen mit kleinem Auftragsvolumen (so genannter Long Tail).

Pull- und Push-Informationen dienen als Orientierungsdaten für die Prozess-Steuerung. Der Wertschöpfungsprozess erstreckt sich über den gesamten Lebenszyklus der Sach- und Dienstleistungen: Er umfasst etwa bei einer Software auch das produzentenseitig ,gepushte“ ReleaseManagement sowie das durch Fehlerrückmeldungen von Usern induzierte technische Änderungsmanagement.

Der Start und Verlauf eines jeden Wertschöpfungsprozesses wird durch zwei Kategorien von Steuerungsinformationen bestimmt: durch Bereitstellung und/oder Bedarf. Als erfolgskritisch für das Zustandekommen eines Bedarf-Bereitstellung-Fit erweisen sich die Induktionsprozesse zwischen diesen Parametern. Im Mittelpunkt stehen dabei die wechselseitigen Induktionsprozesse zwischen Bedarf und Bereitstellung, wenn ein Bedarf ein Angebot oder ein Angebot (s)einen Bedarf induziert. Hinzu kommen Bedarf-Bedarf-Induktionen (z. B. Software induziert einen Wartungsbedarf) und Bereitstellung-BereitstellungInduktionen, z.B. das kombinierte Angebot von Smartphones und Apps.

Push- und Pull-Informationen findet man als Bausteine von zwei Modellansätzen, die sich signifikant durch die jeweiligen Steuerungsobjekte und objektfokussierten Steuerungsinformationen unterscheiden: Koordinationsmodelle steuern Interaktionen zwischen zwei oder mehr Wertschöpfungsakteuren. Solche Aktion-Reaktion-Zusammenhänge (z. B. Kommunikation, Verhandlung, Transaktion) finden in einer Interaktionsarena statt und dienen der
Verhaltenssteuerung. Dabei werden die einzelnen Wertschöpfungsarenen anhand der jeweils involvierten Interaktionsparteien charakterisiert, etwa die ProduzentKunde-Arena. Das Steuerungsinstrumentarium muss an Fähigkeiten und Bereitschaft als den beiden generischen Determinanten von Verhalten ansetzen: Bekanntlich scheitern beispielsweise Innovationen nichts nur an Wissensbarrieren, sondern auch an Willensbarrieren. Deshalb werden Instrumenten-Mixe benötigt, innerhalb derer die informationelle Steuerung durch eine motivationale Steuerung flankiert wird. Zusätzlich zur informationellen Verhaltenssteuerung über Angebote, Bedienungsanleitungen, Aufträge, Anfragen und Beschwerden werden Konditionen (z.B. Leasing- und Flatrate-Modelle), Anreize (z.B. Einführungsrabatte) und Sanktionen (z. B. Vertragsstrafen bei Lieferverzögerungen) eingesetzt, damit es zu einer Übereinstimmung von Bedarf und Bereitstellung kommt. Innerhalb von Produktionsmodellen dienen PushPull-Mechanismen nicht der Verhaltenssteuerung, sondern der Steuerung von Entwicklungs-, Produktions-, Diffusions- und Logistikprozessen über mehrere Wertschöpfungsstufen. Die informationelle Steuerung im Rahmen von Produktionsmodellen bedient sich Informationen über Produktmerkmale (z.B. Funktionalitäten, Qualität, Mengen, Stücklisten) sowie Auslieferungsorte und -termine.

\subsection{Performanz der Wertschöpfungssteuerung}

Zur Messung der Performanz kommen Kriterien der Effektivität und der Effizienz von Wertschöpfungsprozessen zum Einsatz (Timsit et al. 2015). Die Effektivität erfasst den Grad des Fit zwischen Bedarf und Bereitstellung. In Koordinationsmodellen gilt es, mit den Mitteln der Verhaltenssteuerung einen Fit zwischen Wertschöpfung einerseits und Wertschätzung (Nutzen für den Abnehmer) andererseits herzustellen. Hier spielen neben den unterschiedlichen Dimensionen des Wertschöpfungsergebnisses (Funktionalität, Qualität, Quantität, zeitliche und räumliche Verfügbarkeit, Rechtzeitigkeit, Pünktlichkeit) auch Preise eine Rolle: So ist beispielsweise eine Online-Kommunikation mit weniger Gebühren verbunden als eine postalische Kommunikation. Analog rechtfertigt die höhere Wertschätzung für eine Express-Lieferung anstelle einer Standardauslieferung einen höheren Preis. Wie im Fall von EAutomobilen behindert umgekehrt ein zu hoher Preis die Akzeptanz einer Produktinnovation. Die typischen Misfits in Wertschöpfungssystemen in Gestalt von Flops, Ladenhütern, Retouren, Overengineering, Unzuverlässigkeit 
(Qualitätsdefekte, Server-Ausfall), Unterversorgung sowie Diskrepanzen bezüglich der Funktionalität (z. B. ambulante versus stationäre Versorgung) und Quantität (z.B. Mindestabnahmemengen, Mindestvertragslaufzeiten von Service-Verträgen) sind also sowohl das Resultat unzureichender Kommunikation als auch unattraktiver Preispolitik.

Effizienzkriterien erfassen, wie schnell (Zeiteffizienz) und wie kostengünstig (Kosteneffizienz) ein Fit zustande kommt. Ineffizienzen haben zur Folge, dass Manpower, Budgets und andere Ressourcen nicht für die intendierte Wertschöpfung genutzt werden können, weil sie für Anpassungs- und Abstimmungsprozeduren benötigt werden. Die Kosteneffizienz von Produktionsprozessen hängt auch von den eingesetzten Prozesstechnologien ab, etwa von 3D-Druck (additive manufacturing) und Virtual Reality oder Augmented Reality in der Test- und Erprobungsphase (z. B. Simulation, virtuelle Crash-Tests). Analog variiert die Höhe der Koordinationskosten mit der Tragfähigkeit der jeweiligen Koordinationsinfrastruktur (z.B. Vertrauensbasis, Verfügbarkeit von neutralen Drittparteien), weil diese die Dauer von Vertragsverhandlungen oder die Häufigkeit von Rechtsstreitigkeiten beeinflusst.

Das Performance-Management setzt an den Performanz-Determinanten an. Hier konkurrieren zwei Paradigmen der Erzielung eines Fit: Nach dem Verständnis des Abstimmungsparadigmas bildet ein Fit das Ergebnis einer wechselseitigen Anpassung der Vorstellungen auf der Bedarfsseite und auf der Bereitstellungsseite, die sich etwa in Hybrid-Konzepten (z. B. die Hybrid Cloud als Kompromiss von Public- und Private Cloud) oder Service Level Agreements niederschlägt. Gemäß dem Durchsetzungsparadigma kommt ein Fit dadurch zustande, dass sich ein Impuls durchsetzt, d.h. dass sich die Bedarfsseite oder die Bereitstellungsseite einseitig anpasst: Zur Durchsetzung einer Push-Innovation werden beispielsweise Instrumente des Einstellungswandels eingesetzt, etwa das Unfreezing, Moving und Refreezing von Einstellungen zum Cloud Computing, bargeldlosen Zahlungsverkehr oder zur Selbstbedienung. Dabei ist zu beachten, dass die Performanz nicht nur durch die initialisierenden Impulse, sondern auch durch Kontextgegebenheiten determiniert wird. Aus der Perspektive des Chancen-Managements fungieren etwa digitale Prozesstechnologien, z. B. Web 1.0-Instrumente wie Mediatheken, Sendungsverfolgung oder Beschwerdesysteme, Web 2.0-Instrumente in Form von Blogs oder Social Media sowie technische Standards (z. B. Tastaturbelegungen) als Enabler für das Zustandekommen eines Fit. Andere Kontextfaktoren stellen hingegen ein Risikopotenzial dar, weil sie als „Disabler“ oder Inhibitoren einen Fit behindern oder verzögern. Dies betrifft sowohl exogene
Risiken (z. B. Gesetzesänderungen, Streiks, Naturkatastrophen) als auch endogene Risiken, die aus dem Verhalten von Wertschöpfungsakteuren resultieren. Nicht selten entstehen solche Verhaltensrisiken durch Trägheit, Pfadabhängigkeit und konservative Einstellungen.

\subsection{Ablauf- und Aufbauorganisation der Steuerung}

Die beiden Kernfunktionen der Steuerung, Diagnose (Intelligence) und Intervention, liefern die erforderlichen Steuerungsinformationen in Form des Integrationsbedarfs und der Integrationsinstrumente. Aufgabe der Diagnose ist zum einen die Ermittlung der Integrationslücke zwischen Bedarf und Bereitstellung: Sie hängt von zwei Determinanten ab. Zum einen von der Diskrepanz zwischen Impuls und Kontext, etwa davon, wie weit Lieferterminvorstellungen von Produzent und Kunde auseinander liegen. Treten mehrere Push- und oder Pull-Impulse auf, ist deren Kompatibilität zu ermitteln. Zum anderen befassen sich die diagnostischen Aktivitäten mit den jeweiligen Stärken der involvierten Kräfte, z.B. der Stärke des Push-Impulses in Relation zu der Verfestigung des Status-quo oder zu anderen Push-Impulsen. Anhand dieser Informationen lässt sich abschätzen, wie stark ein Parameter die Gegenseite beeinflussen kann bzw. wie groß die Einigungsspielräume für eine wechselseitige Anpassung ausfallen. Darüber hinaus liefert die Diagnose Anhaltspunkte für die Beurteilung der Kosten und Zeiträume, die für eine Abstimmung von Bereitstellung und Bedarf zu erwarten sind. Handelt es sich um gleich stark ausgeprägte Kräfte, ist mit tendenziell höheren Integrationskosten und längeren Integrationszeiten zu rechnen.

Alle Interventionsmaßnahmen bezwecken die Erzielung eines Fit durch Einsatz von Steuerungsinstrumenten. Diese können an zwei Punkten ansetzen: Einerseits kommt eine Modifikation der Stärken von Impulskräften und Kontextkräften in Betracht, um dadurch die Kräftesymmetrie oder -asymmetrie zu verändern. Gemäß dem Durchsetzungsparadigma geht es um die Verstärkung des Impulses (z.B. durch Argumente, Anreize) und/ oder um die Abschwächung des Widerstands. Andererseits muss das Kopplungsmuster zwischen zwei Impulsen, etwa Pull- und Push-Impuls, festgelegt werden. Im Fall der sequentiellen Kopplung kommen die Impulse in unterschiedlichen Etappen oder Phasen eines Wertschöpfungsprozesses zum Einsatz. Bei simultaner Push-Pull-Kopplung müssen die Interventionsmaßnahmen einen Kompromiss bewerkstelligen, wenn zwischen den beiden Impulsen eine Diskrepanz diagnostiziert wurde. 
Sowohl die Aktivitäten der Diagnose als auch der Intervention können auf der Basis unterschiedlicher Partizipationsgrade der betroffenen Wertschöpfungsakteure vonstattengehen. Bei der Beobachtung von Kaufgewohnheiten, Browser-Historien, der Auswertung von Smartphone-Ortungen und dem Erkennen von Präferenzen und Kaufgewohnheiten des „gläsernen Kunden“ über Dataund Web-Mining-Prozeduren oder Predictive AnalyticsTools liegt ein Null-Partizipationsgrad vor. Ein relativ geringer Partizipationsgrad kennzeichnet die kommunikationsbasierte Steuerung, bei der ein Informationsaustausch über eine Verfolgung der Nutzung von Kunden-, Bonus- oder Rabattkarten, über (Double-)Opt-In-basierte Cookies, Kundenbefragungen, Fokusgruppen und Beschwerdesysteme erfolgt. Die Partizipation im Rahmen einer konsensbasierten Steuerung erstreckt sich etwa auf Rahmenlieferverträge und Vertraulichkeitsvereinbarungen. Den höchsten Partizipationsgrad besitzt die kollaborative Steuerung. Hier wird eine kollektive Trägerschaft installiert, die sich in einem Pooling von Ressourcen (z. B. Manpower, Know-how, Wissen) niederschlägt.

\section{Push-Pull-Steuerungsmodelle: Entwicklungsstand}

\subsection{Modell-Architektur}

In den vorliegenden Modellansätzen bewerkstelligen einfache Mono-Impuls-Konstellationen in der Kunde-Produzent-Wertschöpfungsarena (Busines-to-Consumer oder Business-to-Business) dadurch einen Fit von Bedarf und Bereitstellung, dass sich etwa auf einem Verkäufermarkt ein Angebot seine Nachfrage oder auf einem Käufermarkt der Bedarf ein Angebot schafft. Typisch für die kombinierte Steuerung durch Push- und durch Pull-Informationen ist die sequentielle Kopplung der beiden Impulse. Die vorliegenden Push-Pull-Doppelimpuls-Modelle zur operativen Wertschöpfungssteuerung liefern primär eine Typologie der sequentiellen Kopplung von zwei Steuerungsinformationen: Die prognosebasierte Steuerung durch den Produzenten (Push) und die Auftragssteuerung (Pull) durch den Kunden (Reiss 2014). Die einzelnen Typen unterscheiden sich dabei nach den Proportionen von Push und Pull und folglich nach der Positionierung des so genannten Kundenauftragsentkopplungspunkts (Push-PullBoundary), bei dem die Push-Steuerung durch eine PullSteuerung abgelöst wird (Corsten; Gössinger 2001). So erstreckt sich in den so genannten Make-to-order-Ansätzen die Steuerung durch den Kundenauftrag auch auf die
Komponentenherstellung. Im Gegensatz dazu beschränkt sich in den Make-to-stock-Ansätzen der Kundeneinfluss auf die Bestellung aus einem Katalog. Bei strategischen Push-Pull-Wertschöpfungsprozessen ist häufig die PullSteuerung durch Endkunden auf die Front-End-Phase eines Innovationsprozesses beschränkt, während die Produktentwicklung durch Push-Informationen des Herstellers (d.h. Konstruktionspläne, Rezepturen, Meilensteinpläne) gesteuert wird.

Lediglich in rudimentärer Form werden in konventionellen Steuerungsansätzen auch weitere Komplexitätsfaktoren berücksichtigt: So kommen dort neben den originären Impulsen auch derivative Impulse zum Einsatz. Aus den Primärbedarfen abgeleitete Sekundärbedarfe (z.B. Reifen für einen Pkw) stellen insofern „prozessinitialisierende“ Pull-Impulse dar, als sie einen eigenen, vom originären Impuls abweichenden Wertschöpfungscontent betreffen (z. B. Komponenten statt Produkt). Mit den auf den Beschaffungsmarkt gerichteten sekundären Pull-Informationen soll nicht zuletzt der so genannte PeitschenschlagEffekt bekämpft werden (Corsten; Gössinger 2001). Dieser schlägt sich in einem Misfit zwischen Bedarfs- und Bereitstellungsmengen nieder, der sich über die Wertschöpfungsstufen vom Modulzulieferer zum Teilezulieferer vergrößert. In Modellen der so genannten mehrseitigen Märkte - z.B. Medien, die sich sowohl an den Markt der Content-Nachfrager, also Leser, Zuschauer oder InternetUser, als auch der Werbetreibenden (z. B. Annoncen, Banner-Werbung) wenden, werden ebenfalls zusätzliche Komplexitätsaspekte in Wertschöpfungssystemen berücksichtigt (Reiss; Günther 2010). Schließlich decken die Modelle der kompetitiven Auftragsvergabe (über Ausschreibungen und Ideenwettbewerbe) insofern komplexe informationelle Steuerungskonstellationen ab, als zur Lösung eines Problems nicht nur eine Problemlösung, sondern mehrere Lösungsideen vorgelegt werden.

\subsection{Komplexitätsdefizite}

Unter den diversen Schwachstellen vorliegender Modelle zeichnet sich eine Gemeinsamkeit ab: Sie klammern durchweg verschiedene Facetten der real existierenden Komplexität von Wertschöpfung und Steuerung aus. In Anlehnung an die durch Big Data verursachten Herausforderungen lassen sich Komplexitätsdefizite auf den vier Dimensionen Vielzahl (Volume), Vielfalt (Variety), Vieldeutigkeit (als Kehrwert von Veracity/Validity) und Veränderlichkeit (Velocity/Volatiliy) diagnostizieren (Hunt, 2014). Zwischen diesen Dimensionen findet eine Komplexitätsfortpflanzung statt: Vielzahl erzeugt Vielfalt und die- 
se wiederum ist eine Ursache von Vieldeutigkeit und Veränderlichkeit. So signalisiert beispielsweise der Zielkonflikt zwischen effektiver und effizienter Wertschöpfung eingefangen in der bekannten „Quick but dirty“-Formel -, dass mit der Vielfalt (Gegensätzlichkeit) von Zielen auch die (eindeutige) Orientierungsfunktion des Zielsystems verloren gehen kann, weil dieses entgegengesetzte Orientierungen vorgibt.

Begrenzte Vielzahl: Mit der (numerischen) Beschränkung auf eine einzige Wertschöpfungsarena (ProduzentKunde) sowie mit den typischen Mono-Impuls- oder BiImpuls-Ansätzen bleiben alle Multi-Pull- und/oder PushKonstellationen unberücksichtigt. Darüber hinaus fokussieren die Modelle jeweils nur ein oder zwei Wertschöpfungsmerkmale, strategisch meist die Funktionalität, operativ Mengen und Termine. Schließlich bildet die Produktebene eines Wertschöpfungssystems das dominante Steuerungsobjekt, wodurch die Steuerung von Wertschöpfungsprozessen sowohl auf der Ebene der Leistungskomponenten als auch der Leistungskonfigurationen ausgeklammert wird.

Eingeschränkte Vielfalt: Die Kopplung von Push- und Pull-Impulsen erfolgt primär in einem sequenziellen Muster, obwohl in der Realität auch simultane oder subsidiäre Kopplungen existieren. Da jedem Akteur nur eine Rolle zugeordnet wird, bleiben ferner Doppelrollen unberücksichtigt. Hierzu zählen auch hybride Rollenkombinationen, vor allem als Auftraggeber und als RessourcenProvider, was für Modelle des Co-Producing und der CoCreation typisch ist (Reichwald; Piller 2009).

Unterschätzte Vieldeutigkeit: Einige Informationen wie z. B. Kundenaufträge werden als eindeutig und sicher eingestuft, obwohl auch Kunden ihre Bedarfssituation nicht sicher einschätzen können, weshalb etwa Hypes oder kurzlebige Modetrends möglicherweise als nachhaltige Entwicklungen verkannt werden. Auf der Angebotsseite ist häufig unsicher, ob beispielsweise die Produktentwicklungsprojekte rechtzeitig zur geplanten Produktpräsentation auf einer Messe abgeschlossen sind. Auch die Rollen von Wertschöpfungsakteuren sind faktisch durch eine Unschärfe geprägt: Bei einigen Wertschöpfungsakteuren lässt sich nicht klar entscheiden, ob sie Partner oder Konkurrenten sind, was gemeinhin als Coopetition bezeichnet wird (Gast et al. 2014): Das verbreitete Modell der präkompetitiven Zusammenarbeit signalisiert beispielsweise, dass über den Lebenszyklus eines Produkts ein geplanter Rollenwechsel vom Partner zum Wettbewerber stattfindet.

Vernachlässigte Veränderlichkeit: Kaum Untersucht wird in den vorhandenen Steuerungsmodellen, dass sich die Steuerungsinformationen im Zeitablauf mitunter abrupt ändern können. Die Software-Entwicklung wird nicht selten mit kurzfristigen Modifikationen der Lastenhefte und Requirements durch den Kunden konfrontiert. Dieses Kundenverhalten ist möglicherweise eine Reaktion auf unerwartete Kontextveränderungen, etwa Embargos, Naturkatastrophen oder den sprunghaften Technologiewandel (schöpferische Zerstörung). Ferner ändern Wertschöpfungsakteure ihre Rolle im Wertschöpfungssystem: So wird (durch eine so genannte Rückwärtsintegration) aus einem Kunden ein Konkurrent, etwa wenn ein OnlineHändler wie Amazon nicht nur E-Books vertreibt, sondern einen eigenen E-Book-Reader entwickelt.

Wie sich diese Defizite der durch die gängigen PushPull-Steuerungsmodelle betriebenen Komplexitätshandhabung beheben lassen, wird im folgenden Abschnitt anhand von vier Varianten von komplexitätsgerechten Modellansätzen illustriert.

\section{Komplexe Push-Pull- Steuerungsmodelle}

\subsection{Mehrebenen-Architekturen von Wertschöpfungssystemen}

In herkömmlichen Steuerungsansätzen fungiert ein Pro$d u k t$, also eine Sach- oder eine Dienstleistung, als Bezugseinheit der Wertschöpfungssteuerung, etwa der Entwicklung von Computerspielen. Da der Kundennutzen häufig nicht durch einzelne Leistungen, sondern nur durch Kombinationen komplementärer Leistungen erzeugt wird, werden auch diese Leistungskombinationen (z. B. Spielkonsole und Spiele, Pauschalreisen, Einbauküchen, Systeminnovationen wie z. B. die E-Mobilität) zum Objekt der Steuerung. Nicht nur für die bedarfsseitigen, auch für die bereitstellungsseitigen Leistungskombinationen werden komplexe Steuerungsmodelle benötigt. Hierzu zählen etwa Komplementaritäten zwischen Produkt- und Prozessinnovation, z.B. Massenprodukte und Roboterisierung. Komplexitätssteigernd wirkt auch die Tatsache, dass ein Produktionsprozess mitunter zwei (oder mehr) Leistungen hervorbringt, wie z.B. bei der Kraft-Wärme-Kopplung. Schließlich bilden auch Komponenten von Sachleistungen oder Dienstleistungen relevante Wertschöpfungsobjekte, vor allem vor dem Hintergrund der Trends zur Modularisierung, etwa von ERP-Software. Hier reicht das Spektrum von Smartphone-Modulen, etwa Betriebssysteme, Akkus und Klingeltöne, bis zu Vertrags- und Textbausteinen. Die gleichzeitige Steuerung von Produkt- und KomponentenEbene ermöglicht innovative Muster für eine Impuls-Kopplung: So fungiert die Pull-Steuerung auf der Produktebene 
kombiniert mit einer Push-Steuerung auf der Komponentenebene als geeignete Basis für eine individualisierte Massenproduktion. Mit einer solchen Strategie der Mass Customization (Reichwald; Piller 2009) kann man Bereitstellung und Bedarf deshalb gut in Einklang bringen, weil diese komplexe Zweiebenen-Steuerung gleichzeitig für Kosten- und für Kundenorientierung sorgt und damit kundengerechte Produkte zu günstigen Preisen hervorbringt.

\subsection{Erweitertes Spektrum von Wertschöpfungsarenen}

An einem Wertschöpfungsprozess sind nicht nur zwei Akteure in der Rolle des Produzenten und des Kunden beteiligt. Die Relevanz anderer Arenen und damit die Ergänzungsbedürftigkeit der Produzent-Kunde-Arena zeigen sich anhand folgender typischer Erscheinungsformen suboptimaler Performanz der Wertschöpfung: Lieferengpässe auf dem Beschaffungsmarkt im Gefolge der Ausübung von Verkäufermacht durch große Lieferanten verhindern oder verzögern ein bedarfsgerechtes Angebot. Ferner haben bessere Konkurrenzangebote zur Folge, dass der Kunde vorhandene Escape-Optionen in den vertraglichen Vereinbarungen (z. B. über Stornierung) nutzt. Schließlich kann die unsichere Verfügbarkeit komplementärer Sach- und Dienstleistungen, z.B. HD-TV-Geräte ohne HD-TV-Inhalte oder Software ohne Wartung, das Interesse an einem Produkt erheblich reduzieren.

Ergänzt man deshalb die Lieferanten als Wertschöpfungsakteure in der so genannten Supply Chain, die Anbieter komplementärer Leistungen (Komplementoren, Reiss; Günther 2010a) und die Konkurrenten des Produzenten, ergibt sich eine Konfiguration aus fünf Clustern von Wertschöpfungsakteuren, innerhalb derer der Produzent als Bezugseinheit fungiert (Günther 2015). In diesem Value Net erfolgt die Wertschöpfung in vier bilateralen Interaktionsarenen, was sich anschaulich anhand von ELearning illustrieren lässt (Steffens 2015): Hier agiert ein Anbieter von E-Learning-Software (z.B. Adobe Captivate) als Bezugsakteur, andere E-Learning-Produzenten (z.B. Articulate Storyline) als Konkurrenten, Personalentwicklungsabteilungen oder Studenten als Kunden, Autoren und Entwickler von Learning Management-Systemen als Content- bzw. Tool-Lieferanten sowie Anbieter von Virtual Private Network-Infrastrukturen als Komplementoren.

\subsection{Bifunktionale Involvierung von Akteuren}

Die monofunktionale Involvierung des Kunden als Auftraggeber ignoriert die Doppelrolle, die häufig von Kunden im Speziellen und von allen Kategorien von Wertschöpfungsakteuren im Allgemeinen übernommen wird: Sie agieren sowohl als Auftraggeber, der mit seinen Pull-Informationen den Auftragserfüllungsprozess steuert, als auch als Provider, der eigene materielle und immaterielle Ressourcen in den Produktentstehungsprozess einbringt. Im Rahmen einer derart bifunktionalen Involvierung artikulieren Kunden einerseits ihre Forderungen, etwa in Gestalt von Lastenheften, Bedarfsmengen und Terminen. Andererseits speisen sie Informationen (z.B. Konstruktionsdaten), Ideen, Know-how, Manpower, Finanzmittel und Erfahrungen in die F\&E- und Produktionsprozesse ein, z. B. als Pilotkunden oder „Testpiloten“ (etwa in Beta-Programmen) in gemeinsamen Erprobungsaktivitäten, womit sie den Wertschöpfungsprozess fördern.

Diese hybride Involvierung als „Prosumer“, „Produser“ oder „Co-Creator“ hat Konsequenzen sowohl für die Interaktionen als auch die Qualität der Steuerungsinformationen: Die Interaktion zwischen Hersteller und Kunde wandelt sich von verdeckten Screening-Aktivitäten und standardisierten Kommunikationsprozessen (z. B. Bewertungen, Fehlermeldungen, Beschwerden) zu kollaborativen Problemlösungsprozessen. Für das Management der vielfältigen exogenen und endogenen Risiken in einem Wertschöpfungsprozess (z. B. Bestände, Fehlmengen, Retouren, Fehlentwicklungen) eröffnet ein derart hoher Partizipationsgrad neue Optionen: Der Beitrag zum präventiven, also ursachenfokussierten Risiko-Management besteht darin, dass sich die Qualität von Prognosen verbessert, wodurch sich Diskrepanzen zwischen Bereitstellung und Bedarf zwar selten vermeiden, aber zumindest verringern lassen. Auch für das wirkungsfokussierte Management der verbleibenden Risiken liefert eine partizipative Involvierung wertvolle Beiträge: Hier verlagern sich die Strategien der Risikohandhabung von der Schadensüberwälzung (auf eine Partei) auf die Schadensteilung (Risk-Sharing) zwischen beiden Parteien.

\subsection{Multi-Impuls-Konstellationen}

Zwar können Wertschöpfungsprozesse durch einen einzigen Impuls, also Push oder Pull, initialisiert werden. Jedoch münden die komplexen Ebenen- und Arenen-Architekturen von Wertschöpfungsprozessen faktisch eher in Multi-Impuls-Konstellationen. Die Multi-Impuls-Konstellationen verursachen eine erhebliche Steigerung der 
Komplexität von Wertschöpfungsprozessen, was bereits anhand von Doppelimpuls-Konstellationen erkennbar wird: Parallele Doppel-Push-Innovationen auf den Gebieten Akku-Technologie und Betriebssysteme liefern beispielsweise die Technologiebasis für digitale Mobilgeräte. Auf einer Doppel-Pull-Konstellation beruhen z.B. die so genannten Lieferabrufsysteme in der Produzenten-Lieferanten-Arena: Sie koppeln die (monatlichen) Lieferabrufe mit (täglichen) Feinabrufen, um dadurch die Übereinstimmung von Bedarfs- und Bereitstellungsmengen zu verbessern.

Eine erfolgskritische Entwicklungsperspektive der Push-Pull-Steuerungsmodelle ist die Erweiterung um zusätzliche Wertschöpfungsarenen aus dem Value Net. Auf dieser Basis lässt sich ermitteln, welche Auswirkungen die aus mehreren Wertschöpfungsarenen stammenden MultiImpuls-Konstellationen insgesamt auf die Bemühungen um einen Fit von Bedarf und Bereitstellung in der Produzent-Kunde-Arena haben.

Im Rahmen eines sich auf eine erweiterte Wertschöpfungsarena aus Produzent, Kunde und Konkurrenten erstreckenden Steuerungsansatzes lässt sich eine robuste Versorgung mit der erforderlichen Bereitstellungsmenge durch einen Doppel-Pull-Strategie bewerkstelligen: Hier verpflichtet der Kunde (originärer Pull) einen Produzenten, geeignete Lizenznehmer unter seinen Konkurrenten $\mathrm{zu}$ finden (derivativer Pull). Dadurch verringert sich sowohl das Versorgungsrisiko als auch die Abhängigkeit von einer einzigen Bezugsquelle.

Wenn Kundenbedarfe nur durch Leistungsbündel aus mehreren Komplementen befriedigt werden können, ist eine Erweiterung auf eine Produzent-Kunde-Komplementor-Arena erforderlich, die ebenfalls Hand in Hand geht mit einer Vervielfältigung von Steuerungsimpulsen. Hier kann beispielsweise ein Doppel-Pull von Produzent und von Kunde für das fehlende Angebot von Komplementen (z.B. von Ladestationen für E-Fahrzeuge) sorgen bzw. dessen Bereitstellung beschleunigen. Umgekehrt ist ein kombinierter Produzent-Komplementor-Push in der Lage, Widerstände beim Kunden zu überwinden, was dem innovativen Produzenten allein nicht gelungen wäre: Wenn ELearning-Angebote (Push-Informationen) auf Akzeptanzprobleme bei den (konservativen) Kunden stoßen, erweist sich ein Pull-Impuls an die Adresse von komplementären Anbietern im Präsenzlern-Bereich als hilfreich. Ein dadurch bewerkstelligtes kombiniertes Blended LearningAngebot (Steffens 2015) überwindet die Widerstände des Kunden unter der Voraussetzung, dass E-Learning- und Seminar-Inhalte sich optimal ergänzen. Auch das komplementäre Angebot von Zertifizierungen von Produkten (und/oder Anbietern) fördert die Bemühungen um Akzep- $\operatorname{tanz}$ auf der Kundenseite. Dabei wird die Schwäche eines Impulses auf der Produktebene durch Steuerungsimpulse auf der Ebene der Leistungsbündel (Produkt und Gütesiegel) kompensiert.

\section{Zusammenfassung und Ausblick}

Mit den dargestellten Erweiterungen kann man die Bemühungen um eine erfolgreiche Wertschöpfung, sprich um einen höheren Grad der Übereinstimmung zwischen Bereitstellung und Bedarf in Wertschöpfungssystemen, signifikant verbessern. Insbesondere gelingt es, bislang ausgeklammerte, aber real existierende Komplexitätsaspekte $\mathrm{zu}$ berücksichtigen. Allerdings lassen sich dadurch nicht alle aus der Komplexität resultierenden Herausforderungen meistern. Für die deshalb erforderliche Weiterentwicklung des hier skizzierten Ansatzes zeichnen sich zwei Szenarien ab, die sich jeweils an gegensätzlichen Leitbildern der Komplexitätshandhabung orientieren:

Im Verfeinerungsszenario erfolgt eine Steigerung der Modellkomplexität dadurch, dass die Palette der Wertschöpfungsarenen und damit der möglichen Quellen von Wertschöpfungsimpulsen erweitert und differenziert wird (Günther 2015). Dies gelingt durch eine a) Interpolation, wodurch man den Wertschöpfungsbeitrag von Intermediären, etwa Broker oder Cybermediäre (z. B. Betreiber von Suchmaschinen und Innovationsplattformen) erfassen kann, b) Intrapolation, womit sich etwa innerhalb der Kundenarena die Beziehungen zwischen einzelnen Kunden für ein Word-of-Mouth-Marketing nutzen lassen, sowie durch eine c) Extrapolation: Das sich bei den (komplementären) Gütesiegeln und Zertifikaten abzeichnende inflationäre Angebot erfordert zusätzliche Komplementoren, mit deren Hilfe sich die „Qualität der Qualitätssiegel“ prüfen lässt.

Im Entfeinerungsszenario findet hingegen eine radikale Komplexitätsreduktion dadurch statt, dass man auf eine Differenzierung von Wertschöpfungsarenen und -rollen verzichtet. Dieser Ansatz stellt eine konsequente Weiterentwicklung der bifunktionalen Involvierung von Wertschöpfungsakteuren dar. Alle Akteure übernehmen Doppel-Rollen als mögliche Quellen von Push- und von PullImpulsen. Dieses Rollenkonzept liegt den hochgradig interaktiven Ansätzen von Business Ecosystems, Democratic Innovation, Communities of Innovation, Crowdsourcing und Open Innovation zugrunde (Santos 2015). Die dabei praktizierte Share Economy (Leitbild: „Teilen statt Besitzen") geht einher mit dem partizipativen Teilen von Chancen und Risiken der Wertschöpfungsprozesse. Allerdings werden dadurch primär die Ideengenerierung und Akzeptanz innerhalb der Community (z.B. von Open Source- 
Software wie Apache Open Office) unterstützt, ohne dass die Akzeptanz außerhalb der Community gesichert wäre.

\section{Literatur}

Corsten, Hans; Gössinger, Ralf, 2001. Determinanten zur Bestimmung des Kundenauftragsentkoppelungspunktes in der Supply Chain. In: PPS Management 6 (4), 22-26. ISSN: 1434-2308.

Hunt, David, 2014: Big Data Challenges: volume, variety, velocity \& veracity. Raleigh, NC: Hunt. https://research.ncsu.edu/results/f iles/2014/12/fall-2014-big-data-challenges.pdf [14.02.2016].

Reichwald, Ralf; Piller, Frank T., 2009: Interaktive Wertschöpfung. 2. Aufl. Wiesbaden: Gabler. ISBN 2009. 978-3834909725.

Gast, Johanna et al., 2014. Coopetition research: towards a better understanding of past trends and future directions. In: International Journal of Entrepreneurship and Small Business 24 (4), 492-521. ISSN 1476-1297.

Hagel, John; Brown, John Seeley, 2005: From Push to Pull-Emerging Models for Mobilizing Resources. Hagel; Seeley. http://www.joh nhagel.com/paper_pushpull.pdf [14.02.2016].

Rohweder, Jan P. et al., 2008. Informationsqualität: Definitionen, Dimensionen und Begriffe. In: Hildebrand, Knut et al., 2008: Daten- und Informationsqualität. 1. Aufl. 25-45. Wiesbaden: Vieweg+Teubner. ISBN 978-3-8348-9953-8.

Di Stefano, Giada; Gambardella, Alfonso; Verona, Gianmario, 2012. Technology push and demand pull perspectives in innovation studies: Current findings and future research directions. In: Research Policy 41(8), 1283-1295. ISSN 0048-7333.

Günther, Armin, 2015: Complementor Relationship Management. 1. Auf. Wiesbaden: Gabler. ISBN 978-3-658-08565-0.

Reiss, Michael, 2014. Push-Pull-Architekturen für das Produktionsmanagement. In: Zeitschrift für wirtschaftlichen Fabrikbetrieb 109 (9), 599-603. ISSN 0947-0085.

Reiss, Michael; Günther, Armin, 2010. Mehrseitige Märkte: Paradigmenwechsel vom Markt- zum Netzwerk-Ansatz. In: WiSt - Wirtschaftswissenschaftliches Studium 39 (4), 176-181. ISSN 0340-1650.
Reiss, Michael; Günther, Armin, 2010a. Outsourcing von Informationstechnologie: Spektrum und Stellenwert von Komplementoren. In: Information. Wissenschaft \& Praxis 61 (1), 45-50. ISSN 1434-4653.

Santos, Antonio B., 2015. Open Innovation research: trends and influences-a bibliometric analysis. In: Journal of Innovation Management 3 (2), 131-165. ISSN 2183-0606.

Simchi-Levi, David et al., 2008. Designing and managing the supply chain. 3. Aufl. Boston, MA: McGraw-Hill Education. ISBN-13: 978-0073341521.

Steffens, Dirk 2015: Blended Learning: Kosteneffiziente Personalentwicklung durch hybride Konfiguration von Präsenzlernen und ELearning 1. Aufl. Berlin: epubli. ISBN 9783737551595.

Tidd, Joe; Bessant, John, 2013. Managing innovation: integrating technological, market and organizational change. 5. Aufl. Chichester: Wiley. ISBN 978-1-118-36063-7.

Timsit, Jean-Philippe et al., 2015. The effect of market-pull vs. resource-push orientation on performance when entering new markets. In: Journal of Business Research 68 (9), 2005-2014. ISSN 0148-2963.

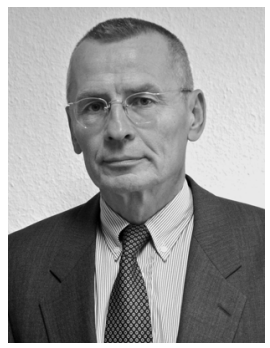

Prof. Dr. Michael Reiss

Lehrstuhl für Organisation Universität Stuttgart

Keplerstraße 17 70174 Stuttgart michael.reiss@bwi.uni-stuttgart.de

Die Forschungs- und Lehrtätigkeit von Michael Reiss umfasst die Gebiete Netzwerkorganisation, strategiegerechte Organisationsgestaltung, Change Management, Unternehmertum, Personal- und Unternehmensführung sowie Projektorganisation. Nach dem Studium der Psychologie und Wirtschaftswissenschaften war er an den Universitäten Freiburg, Köln, Ingolstadt und Stuttgart tätig. 